\title{
Clinical significance of pre-operative neutrophil lymphocyte ratio and platelet lymphocyte ratio as prognostic factors for patients with colorectal cancer
}

\author{
ZHEN-YU ZOU ${ }^{1}$, HAI-LIANG LIU ${ }^{2}$, NING NING $^{3}$, SONG-YAN LI ${ }^{1}$, XIAO-HUI DU ${ }^{1,4}$ and RONG LI ${ }^{1}$ \\ Departments of ${ }^{1}$ General Surgery and ${ }^{2}$ Oncological Surgery, Chinese People's Liberation Army General Hospital, \\ Beijing 100853; ${ }^{3}$ Department of Gastrointestinal Surgery, Peking University International Hospital, Beijing 102206; \\ ${ }^{4}$ Department of General Surgery, Hainan Branch of the Chinese People's Liberation Army General Hospital, \\ Sanya, Hainan 572013, P.R. China
}

Received February 12, 2015; Accepted January 8, 2016

DOI: $10.3892 / \mathrm{ol} .2016 .4216$

\begin{abstract}
The present study aimed to investigate the independent prognostic values of the pre-operative neutrophil lymphocyte ratio (NLR) and platelet lymphocyte ratio (PLR) in patients with colorectal cancer (CRC). The present study retrospectively analyzed the data of 216 patients with CRC from a single hospital. The clinicopathological characteristics of the patients were compared and prognostic factors were evaluated. NLR and PLR were associated with tumor differentiation status and the tumor diameter, respectively, and PLR was also associated with the primary tumor classification (T classification). Furthermore, NLR and PLR were positively associated with each other $\left(\mathrm{R}^{2}=0.5368 ; \mathrm{P}<0.0001\right)$. Univariate analyses indicated that stage II and III patients with a high NLR ( $\geq 4.98 ; \mathrm{P}<0.001)$ or PLR $(\geq 246.36$; $\mathrm{P}<0.001)$ possessed a significantly poorer 5-year OS rate compared with those with a low NLR or PLR. Post-operative adjuvant chemotherapy improved the 5-year OS rate in patients with a high NLR or PLR. Multivariate analyses indicated that NLR and PLR were independent prognostic factors [NLR, relative risk $(R R)=4.074$ and $\mathrm{P}<0.001$; $\mathrm{PLR}, \mathrm{RR}=2.029$ and $\mathrm{P}=0.029$ ] in patients with $\mathrm{CRC}$, and were associated with the $\mathrm{T}$ classification, lymph node metastasis and post-operative adjuvant chemotherapy response of patients. Additionally, the area under the curve (AUC) was 0.748 for NLR (95\% CI, 0.684-0.804; P<0.0001) and 0.690 for PLR (95\% CI, 0.623-0.751; P<0.0001). The RR and AUC indicated that NLR was the superior predictive factor in patients with CRC. In conclusion, the pre-operative
\end{abstract}

Correspondence to: Professor Rong Li or Professor Xiao-Hui Du, Department of General Surgery, Chinese People's Liberation Army General Hospital, 28 Fuxing Road, Beijing 100853, P.R. China

E-mail: lirong@medmail.com.cn

E-mail: duxiaohui301@sina.com

Key words: neutrophil lymphocyte ratio, platelet lymphocyte ratio, colorectal cancer, prognosis
NLR and PLR were significant independent prognostic factors in patients with CRC, and NLR was more effective as a prognostic marker compared with PLR. Adjuvant chemotherapy appeared to be more effective in CRC patients with a higher NLR or PLR.

\section{Introduction}

Colorectal cancer (CRC) is the third most common cancer and the fourth leading cause of cancer mortality according to GLOBOCAN 2012 (1). High tumor stage and histological grade, in addition to the number of resected lymph nodes and intravascular invasion have all been identified as prognostic factors (2). Thus, investigation of the mechanisms underlying CRC etiology is of clinical importance.

The association between inflammation and cancer was identified in the 19th century by Rudolf Virchow (3). Since then, studies have confirmed the broad and significant effect of inflammation on tumor development, progression and response to therapy (4-6). The presence of inflammatory cells, growth factors, activated stroma and DNA-damage-promoting agents in the inflammatory environment function to sustain cell proliferation and increase neoplastic risk (5). Meanwhile, the tumor increases inflammatory process and promotes tumor proliferation and metastases development by decreasing apoptosis and increasing angiogenesis and DNA damage $(4,5)$. Notably, the scope and effects of an inflammatory reaction have always been assessed according to a number of biochemical markers, including the neutrophil lymphocyte ratio (NLR) and platelet lymphocyte ratio (PLR), which are inexpensive markers of systemic inflammation (7). Previous studies have indicated that elevated NLR or PLR suggested a poor prognosis for various types of cancer, including ovarian (8), breast $(9,10)$, non-small cell lung $(11)$, esophageal $(12,13)$, gastric $(7,14)$, hepatocellular (15) and CRC (2,16-18). However, the current literature leaves certain questions unanswered. Firstly, it is unknown as to which parameter is superior for predicting the outcome of CRC. Secondly, to the best of our knowledge, only 2 studies have compared the prognostic value of NLR and PLR in patients with CRC $(17,18)$. One study 
demonstrated that elevated NLR and PLR were identified as significant poor prognostic factors in metastatic CRC, and that NLR led to improved prognostic predictors (17). On the contrary, the other study indicated that PLR was a superior prognostic marker (18). However, the grouping of NLR and PLR in these studies was based on a cut-off value that was calculated in other studies, which did not accurately reflect the data. Therefore, the aim of the present study was to explore the clinical significance of NLR and PLR as independent prognostic factors in patients with CRC and to identify the factor that is more effective in this role.

\section{Patients and methods}

Patients. A retrospective analysis was performed on data from 216 patients with CRC that underwent radical surgery at Chinese People's Liberation Army (PLA) General Hospital (Beijing, China) between July 2006 and June 2012. Patients were selected for the present study according to the following inclusion criteria: CRC confirmed by histopathology; radical resection with microscopically tumor-free resection margins; and complete blood count, clinicopathological and follow-up data. The exclusion criteria excluded patients for the following reasons: Underwent palliative surgery; complications of intestinal obstruction, hemorrhage or enterobrosis resulting in emergency surgery; clinical evidence of infection, systemic inflammation or autoimmune disorder; underwent or accepted neoadjuvant chemotherapy or radiotherapy; succumbed to CRC or other causes within 30 days of surgery; and a history of other malignancies. All enrolled CRC patients were staged according to the American Joint Committee on Cancer tumor-node-metastasis (TNM) classification system (19) and were treated according to the National Comprehensive Cancer Network clinical practice guidelines in oncology for colon (20) and rectal cancer (21). All the patients provided written informed consent prior to the present study, which was approved by the Ethics Committee of Chinese PLA General Hospital.

Follow-up of patients. Letters and telephone interviews were used to follow-up each patient. The last follow-up date was July 1,2013. The overall survival (OS) time was defined as the time between surgery and mortality from any cause or to the last date of follow-up.

NLR and PLR grouping. All blood samples were taken 1 week prior to surgery. NLR was defined as the absolute neutrophil count divided by the absolute lymphocyte count. The patients were divided into two groups based on the NLR value, the NLR $<4.98$ and NLR $\geq 4.98$ groups. PLR was defined as the absolute platelet count divided by the absolute lymphocyte count. The patients were divided into two groups based on the PLR value, the PLR $<246.36$ and PLR $\geq 246.36$ groups.

Statistical analysis. Statistical analysis was performed with IBM SPSS software version 20 (IBM SPSS, Armonk, NY, USA). Receiver operating characteristic (ROC) curves were plotted to calculate the area under the ROC curve (AUC). The Youden index (YI) was calculated to determine the optimal cutoff value for NLR and PLR. Linear regression was

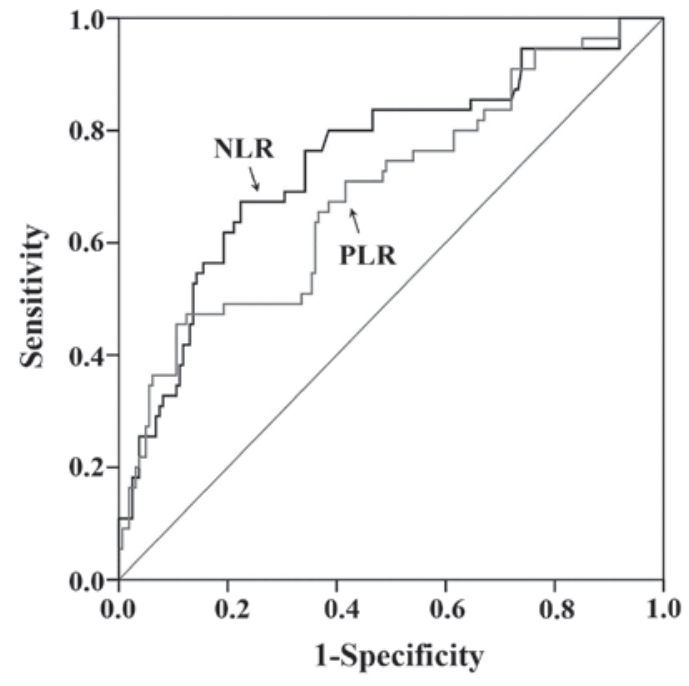

Figure 1. ROC curves for NLR and PLR. The ROC curve for NLR is indicated by the black line, with an AUC of 0.748 (95\% CI, 0.684-0.804; $\mathrm{P}<0.0001)$. The ROC curve for PLR is indicated by the gray line, with an AUC of 0.690 (95\% CI, 0.623-0.751; $\mathrm{P}<0.0001)$. ROC, receiver operating characteristic; NLR, neutrophil lymphocyte ratio; PLR, platelet lymphocyte ratio; AUC, area under the ROC curve; $\mathrm{CI}$, confidence interval.

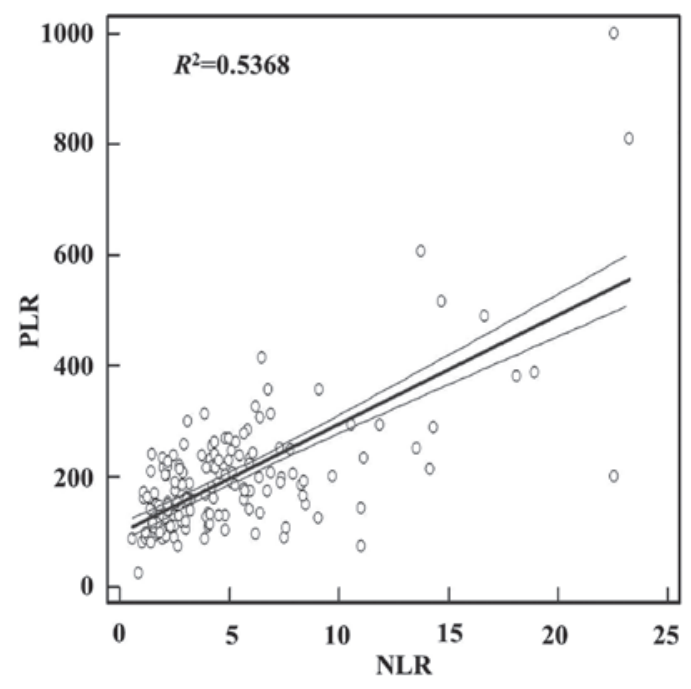

Figure 2. Linear regression of NLR and PLR. NLR and PLR were positively associated with each other. Regression equation: $y=97.3626+19.7032 x$ $\left(\mathrm{R}^{2}=0.5368 ; \mathrm{P}<0.0001\right)$. NLR, neutrophil lymphocyte ratio; PLR, platelet lymphocyte ratio.

performed to evaluate the association between NLR and PLR. Continuous variables are presented as the mean \pm standard error and categorical variables are presented as frequencies and percentages. The differences between clinicopathological characteristics grouped by NLR or PLR were compared using the Pearson $\chi^{2}$ test or Fisher's exact test for categorical variables and Student's $t$-test for continuous variables. Survival time curves and 5-year OS rates were calculated using the Kaplan-Meier method of univariate analysis, and the differences were compared using the log-rank test. Independent prognostic factors were evaluated using the Cox proportional hazard model of multivariate analysis. Variables with a univariate analysis value of $\mathrm{P}<0.05$ were entered into multivariate 
Table I. Clinicopathological characteristics of 216 patients with colorectal cancer, grouped by NLR and PLR.

\begin{tabular}{|c|c|c|c|c|c|c|}
\hline \multirow[b]{2}{*}{ Characteristic } & \multicolumn{3}{|c|}{ NLR } & \multicolumn{3}{|c|}{ PLR } \\
\hline & $\begin{array}{c}\text { NLR }<4.98 \\
\mathrm{n}(\%)\end{array}$ & $\begin{array}{c}\text { NLR } \geq 4.98 \\
\mathrm{n}(\%)\end{array}$ & P-value & $\begin{array}{c}\text { PLR }<246.36 \\
\mathrm{n}(\%)\end{array}$ & $\begin{array}{c}\text { PLR } \geq 246.36 \\
\text { n }(\%)\end{array}$ & P-value \\
\hline Total & $141(100.0)$ & $75(100.0)$ & & $172(100.0)$ & $44(100.0)$ & \\
\hline Gender & & & 0.659 & & & 0.114 \\
\hline Male & $91(64.5)$ & $46(61.3)$ & & $114(66.3)$ & $23(52.3)$ & \\
\hline Female & $50(35.5)$ & $29(38.7)$ & & $58(33.7)$ & $21(47.7)$ & \\
\hline Age, years & $53.90 \pm 12.1$ & $54.49 \pm 10.8$ & 0.239 & $53.81 \pm 11.3$ & $55.27 \pm 13.0$ & 0.145 \\
\hline PLR & $154.94 \pm 57.1$ & $278.89 \pm 162.4$ & $<0.001$ & & & \\
\hline NLR & & & & $3.81 \pm 2.8$ & $10.19 \pm 6.0$ & $<0.001$ \\
\hline Tumor location & & & 0.887 & & & 0.398 \\
\hline Colon & $73(51.8)$ & $40(53.3)$ & & $87(50.6)$ & $26(59.1)$ & \\
\hline Rectum & $68(48.2)$ & $35(46.7)$ & & $85(49.4)$ & $18(40.9)$ & \\
\hline Differentiation & & & 0.019 & & & 0.019 \\
\hline Well & $26(18.4)$ & $5(6.7)$ & & $28(16.3)$ & $3(6.8)$ & \\
\hline Moderate & $83(58.9)$ & $43(57.3)$ & & $104(60.5)$ & $22(50.0)$ & \\
\hline Poor & $32(22.7)$ & $27(36.0)$ & & $40(23.3)$ & $19(43.2)$ & \\
\hline Tumor diameter, $\mathrm{cm}$ & $4.54 \pm 1.9$ & $5.73 \pm 3.1$ & 0.007 & $4.58 \pm 2.0$ & $6.40 \pm 3.4$ & 0.001 \\
\hline T classification & & & 0.187 & & & 0.006 \\
\hline $\mathrm{T} 1+\mathrm{T} 2$ & $23(16.3)$ & $7(9.3)$ & & $28(16.3)$ & $2(4.5)$ & \\
\hline $\mathrm{T} 3$ & $81(57.4)$ & $41(54.7)$ & & $101(58.7)$ & $21(47.7)$ & \\
\hline $\mathrm{T} 4$ & $37(26.2)$ & $27(36.0)$ & & $43(25.0)$ & $21(47.7)$ & \\
\hline $\mathrm{LN}$ metastasis & & & 0.070 & & & 0.058 \\
\hline No & $77(54.6)$ & $36(48.0)$ & & $93(54.1)$ & $20(45.5)$ & \\
\hline N1 & $50(35.5)$ & $23(30.7)$ & & $60(34.9)$ & $13(29.5)$ & \\
\hline $\mathrm{N} 2$ & $14(9.9)$ & $16(21.3)$ & & $19(11.0)$ & $11(25.0)$ & \\
\hline Distant metastasis & & & 0.053 & & & 0.122 \\
\hline M0 & $128(90.8)$ & $61(81.3)$ & & $154(89.5)$ & $35(79.5)$ & \\
\hline M1 & $13(9.2)$ & $14(18.7)$ & & $18(10.5)$ & $9(20.5)$ & \\
\hline TNM staging & & & 0.062 & & & 0.082 \\
\hline $\mathrm{I}$ & $16(11.3)$ & $4(5.3)$ & & $19(11.0)$ & $1(2.3)$ & \\
\hline II & $61(43.3)$ & $28(37.3)$ & & $71(41.3)$ & $18(40.9)$ & \\
\hline III & $53(37.6)$ & $29(38.7)$ & & $66(38.4)$ & $16(36.4)$ & \\
\hline IV & $11(7.8)$ & 14 (18.7) & & $16(9.3)$ & $9(20.5)$ & \\
\hline
\end{tabular}

NLR, neutrophil lymphocyte ratio; PLR, platelet lymphocyte ratio; T, primary tumor; LN, lymph node; TNM, tumor-node-metastasis.

analysis. Each test was two-tailed. $\mathrm{P}<0.05$ was considered to indicate a statistically significant difference.

\section{Results}

The ROC curve for optimal cutoff value and AUC. When the NLR was 4.98, YI was at its maximum (YI=0.4491), demonstrating that 4.98 was the optimal cutoff value for NLR. Therefore, patients were divided into low NLR $(<4.98)$ and high NLR $(\geq 4.98)$ groups. When the PLR was 246.36, YI was at its maximum (YI=0.3490). Therefore, patients were divided into low PLR $(<246.36)$ and high PLR $(\geq 246.36)$ groups.
The AUC for NLR was 0.748 (95\% CI, 0.684-0.804; $\mathrm{P}<0.0001)$ and PLR was 0.690 (95\% CI, 0.623-0.751; $\mathrm{P}<0.0001$; Fig. 1).

Association of NLR and PLR with clinicopathological characteristics. In total, 216 patients were enrolled in the present study. The median follow-up time was 38 months, with a range of 3-85 months. At the final follow-up date, 161 patients (74.5\%) were alive. A comparison between the clinicopathological characteristics and the NLR and PLR are exhibited in Table I. High NLR and PLR were associated with poor tumor differentiation and a larger tumor diameter, respectively $(\mathrm{P}<0.05)$. A high PLR was also associated with a poor primary 
Table II. Univariate analyses in 216 patients with colorectal cancer.

\begin{tabular}{|c|c|c|c|}
\hline Characteristic & $\mathrm{n}$ & 5-year OS rate, $\%$ & P-value \\
\hline Gender & & & 0.724 \\
\hline Male & 137 & 72.4 & \\
\hline Female & 79 & 65.4 & \\
\hline Age, years & & & 0.662 \\
\hline$<50$ & 75 & 71.1 & \\
\hline$\geq 50$ & 141 & 69.0 & \\
\hline NLR & & & $<0.001$ \\
\hline$<4.98$ & 141 & 84.7 & \\
\hline$\geq 4.98$ & 75 & 42.7 & \\
\hline PLR & & & $<0.001$ \\
\hline$<246.36$ & 172 & 79.2 & \\
\hline$\geq 246.36$ & 44 & 34.0 & \\
\hline Tumor location & & & 0.003 \\
\hline Colon & 113 & 62.9 & \\
\hline Rectum & 103 & 77.4 & \\
\hline Differentiation & & & 0.014 \\
\hline Well & 31 & 71.0 & \\
\hline Moderate & 126 & 76.8 & \\
\hline Poor & 59 & 55.0 & \\
\hline Tumor diameter, $\mathrm{cm}$ & & & 0.967 \\
\hline$<5$ & 104 & 68.9 & \\
\hline$\geq 5$ & 112 & 71.0 & \\
\hline T classification & & & $<0.001$ \\
\hline $\mathrm{T} 1+\mathrm{T} 2$ & 30 & 87.7 & \\
\hline $\mathrm{T} 3$ & 122 & 82.6 & \\
\hline $\mathrm{T} 4$ & 64 & 36.1 & \\
\hline LN metastasis & & & $<0.001$ \\
\hline NO & 113 & 86.3 & \\
\hline N1 & 73 & 63.5 & \\
\hline $\mathrm{N} 2$ & 30 & 22.2 & \\
\hline Distant metastasis & & & $<0.001$ \\
\hline M0 & 189 & 76.2 & \\
\hline M1 & 27 & 9.6 & \\
\hline TNM staging & & & $<0.001$ \\
\hline I & 20 & 88.9 & \\
\hline II & 89 & 88.2 & \\
\hline III & 82 & 57.7 & \\
\hline IV & 25 & 0.0 & \\
\hline Chemotherapy & & & 0.047 \\
\hline Yes & 149 & 73.6 & \\
\hline No & 67 & 62.7 & \\
\hline Score & & & $<0.001$ \\
\hline 0 & 133 & 85.5 & \\
\hline 1 & 47 & 60.0 & \\
\hline 2 & 36 & 27.2 & \\
\hline
\end{tabular}

OS, overall survival; NLR, neutrophil lymphocyte ratio; PLR, platelet lymphocyte ratio; T, primary tumor; LN, lymph node; TNM, tumor-node-metastasis.
Table III. Multivariate analyses in 216 patients with colorectal cancer.

\begin{tabular}{lrc}
\hline Feature & P-value & RR (95\% CI $)$ \\
\hline NLR & $<0.001$ & $4.074(1.975-8.405)$ \\
PLR & 0.029 & $2.029(1.077-3.821)$ \\
Tumor location & 0.362 & $0.741(0.389-1.411)$ \\
Differentiation & 0.755 & $0.918(0.538-1.567)$ \\
T classification & $<0.001$ & $3.516(2.008-6.156)$ \\
LN metastasis & 0.030 & $1.830(1.059-3.161)$ \\
Distant metastasis & 0.389 & $1.840(0.459-7.375)$ \\
TNM staging & 0.576 & $1.322(0.497-3.516)$ \\
Chemotherapy & 0.021 & $2.006(1.110-3.625)$ \\
\end{tabular}

RR, relative risk; CI, confidence interval; NLR, neutrophil lymphocyte ratio; PLR, platelet lymphocyte ratio; T, primary tumor; $\mathrm{LN}$, lymph node; TNM, tumor-node-metastasis.

tumor classification ( $\mathrm{T}$ classification) $(\mathrm{P}=0.006)$. In addition, there was a positive association between NLR and PLR. The regression equation was as follows: $y=97.3626+19.7032 x$ $\left(\mathrm{R}^{2}=0.5368 ; \mathrm{P}<0.0001\right.$; Fig. 2$)$.

Association of NLR and PLR with the 5-year OS rate. The 1, 3 and 5-year OS rates were 95.8, 76.0 and 70.2\%, respectively. Univariate analyses demonstrated that the NLR, PLR, tumor location $(\mathrm{P}=0.003)$, tumor differentiation $(\mathrm{P}=0.014)$, $\mathrm{T}$ classification $(\mathrm{P}<0.001)$, lymph node $(\mathrm{LN})$ metastasis $(\mathrm{P}<0.001)$, distant metastasis $(\mathrm{P}<0.001)$, TNM staging $(\mathrm{P}<0.001)$ and administration of post-operative adjuvant chemotherapy $(\mathrm{P}=0.047)$ were associated with the 5-year OS rate (Table II). Patients with a high NLR possessed a significantly poorer 5-year OS rate compared with patients with a low NLR (42.7 vs. 84.7\%; P<0.001; Fig. 3). Patients with a high PLR possessed a significantly poorer 5-year OS rate compared with patients with a low PLR (34.0 vs. $79.2 \%$; $\mathrm{P}<0.001$; Fig. 4).

Patients with high values for NLR and PLR were allocated a score of 2, patients with a high NLR or PLR value were allocated a score of 1 , and patients that did not possess high NLR or PLR values were allocated a score of 0 . Univariate analysis revealed that patients with a score of 2 possessed a significantly poorer 5-year OS rate $(27.2 \%)$ compared with patients with a score of $1(60.0 \%)$ or $0(85.5 \%$; $\mathrm{P}<0.001)$. Patients with a score of 1 had a significantly poorer 5 -year OS rate compared with patients with a score of $0(\mathrm{P}<0.001$; Table II).

Multivariate analyses of independent prognostic factors. Multivariate analyses using the Cox proportional hazards model identified NLR, PLR, T classification $(\mathrm{P}<0.001 ; 95 \% \mathrm{CI}$, 2.008-6.156), LN metastasis ( $\mathrm{P}=0.030 ; 95 \% \mathrm{CI}, 1.059-3.161)$ and post-operative adjuvant chemotherapy $(\mathrm{P}=0.021 ; 95 \% \mathrm{CI}$, 1.110-3.625) as independent prognostic factors (Table III). The risk of succumbing to CRC for patients with a high NLR was $>4$ times higher than patients with a low NLR (relative risk $(\mathrm{RR})=4.074 ; \mathrm{P}<0.001 ; 95 \% \mathrm{CI}, 1.975-8.405)$. The risk of succumbing to $\mathrm{CRC}$ for patients with a high PLR was $>2$ times 
Table IV. The 5-year OS rate for NLR and PLR stratified by TNM staging.

\begin{tabular}{|c|c|c|c|c|c|c|c|c|}
\hline \multirow[b]{2}{*}{ Ratio } & \multicolumn{2}{|r|}{ TNM stage I } & \multicolumn{2}{|r|}{ TNM stage II } & \multicolumn{2}{|r|}{ TNM stage III } & \multicolumn{2}{|r|}{ TNM stage IV } \\
\hline & $\mathrm{n}$ & 5 -year OS rate, $\%$ & $\mathrm{n}$ & 5 -year OS rate, $\%$ & $\mathrm{n}$ & 5 -year OS rate, $\%$ & $\mathrm{n}$ & 5 -year OS rate, $\%$ \\
\hline \multicolumn{9}{|l|}{ NLR } \\
\hline$<4.98$ & 16 & 86.7 & 61 & 95.0 & 53 & 82.0 & 11 & 0.0 \\
\hline$\geq 4.98$ & 4 & 100.0 & 28 & 74.0 & 29 & 15.2 & 14 & 0.0 \\
\hline P-value & & 0.514 & & 0.002 & & $<0.001$ & & 0.338 \\
\hline \multicolumn{9}{|l|}{ PLR } \\
\hline$<246.36$ & 19 & 88.9 & 71 & 94.1 & 66 & 74.2 & 16 & 0.0 \\
\hline$\geq 246.36$ & 1 & NA & 18 & 65.5 & 16 & 7.5 & 9 & 16.7 \\
\hline P-value & & NA & & $<0.001$ & & $<0.001$ & & 0.256 \\
\hline
\end{tabular}

OS, overall survival; NLR, neutrophil lymphocyte ratio; PLR, platelet lymphocyte ratio; TNM, tumor-node-metastasis; NA, not available.

Table V. The 5-year OS rate for adjuvant chemotherapy stratified by NLR or PLR.

\begin{tabular}{|c|c|c|c|c|c|c|c|c|}
\hline \multirow[b]{2}{*}{ Chemotherapy } & \multicolumn{2}{|r|}{ NLR $<4.98$} & \multicolumn{2}{|r|}{ NLR $\geq 4.98$} & \multicolumn{2}{|r|}{ PLR $<246.36$} & \multicolumn{2}{|r|}{ PLR $\geq 246.36$} \\
\hline & $\mathrm{n}$ & 5 -year OS rate, $\%$ & $\mathrm{n}$ & 5 -year OS rate, $\%$ & $\mathrm{n}$ & 5 -year OS rate, $\%$ & $\mathrm{n}$ & 5 -year OS rate, $\%$ \\
\hline Yes & 96 & 87.2 & 53 & 49.1 & 119 & 81.5 & 30 & 42.7 \\
\hline No & 45 & 79.7 & 22 & 29.2 & 53 & 74.1 & 14 & 21.4 \\
\hline $\mathrm{P}$-value & & 0.255 & & 0.015 & & 0.259 & & 0.019 \\
\hline
\end{tabular}

OS, overall survival; NLR, neutrophil lymphocyte ratio; PLR, platelet lymphocyte ratio.

higher than patients with a low PLR ( $R R=2.029 ; \mathrm{P}=0.029$; 95\% CI, 1.077-3.821).

Association between the NLR and PLR and 5-year OS rate stratified by TNM staging. Patients in the NLR and PLR groups were stratified according to TNM staging. The results indicated that patients with TNM stage II or III disease and a high NLR or PLR possessed a significantly poorer 5-year OS rate compared with patients with a low NLR (stage II, $\mathrm{P}=0.002$; stage III, $\mathrm{P}<0.001$ ) or PLR (stage II, $\mathrm{P}<0.001$; stage III, $\mathrm{P}<0.001$; Table IV).

Association between patients grouped by post-operative adjuvant chemotherapy and the 5-year OS rate stratified by NLR or PLR. The patients were divided into two groups according to post-operative adjuvant chemotherapy. Univariate analyses stratified by the NLR or PLR revealed that adjuvant chemotherapy did not affect the 5-year OS rate in patients with a low NLR ( $\mathrm{P}=0.255)$ or PLR $(\mathrm{P}=0.259)$. However, adjuvant chemotherapy increased the 5-year OS rate from 29.2 to $49.1 \%$ in patients with a high NLR ( $\mathrm{P}=0.015$; Fig. 5) and from 21.4 to $42.7 \%$ in patients with a high PLR ( $\mathrm{P}=0.019$; Fig. 6; Table V).

\section{Discussion}

CRC is the third most common cancer and the fourth leading cause of cancer-associated mortality, according to GLOBOCAN 2012 (1). In China, an increase in CRC risk factors, including an aging population and changes in eating habits (increased meat intake and reduced fiber intake), has rendered CRC the fifth most common cancer in the country, following lung, stomach, liver and esophageal cancers (22).

In 1863, Rudolf Virchow hypothesized that the origin of cancer was at sites of chronic inflammation, and this causal association between inflammation, innate immunity and cancer is more widely accepted at present $(3,5)$. Inflammation is significant in tumor development, progression and response to therapy (4). The presence of inflammatory cells, growth factors, activated stroma and DNA-damage-promoting agents in the inflammatory environment act to sustain cell proliferation and increase neoplastic risk (5). In turn, the tumor increases the inflammatory process and promotes tumor proliferation and metastases development by decreasing apoptosis and increasing angiogenesis and DNA damage $(4,5)$. The strongest association of chronic inflammation with malignancy has been reported in CRC arising from inflammatory bowel diseases $(5,23)$. Therefore, the prognosis of CRC is not only associated with the biological behavior of the tumor, but also with the inflammatory reaction of the host.

Inflammatory reactions have always been assessed by a number of biochemical markers, in particular traditional hematological markers, including C-reactive protein (CRP) and differential leukocyte and platelet counts. CRP is an index of systemic inflammation and has been identified as a prognostic factor in patients with CRC and gastric cancer $(24,25)$. However, serum CRP levels are not routinely 


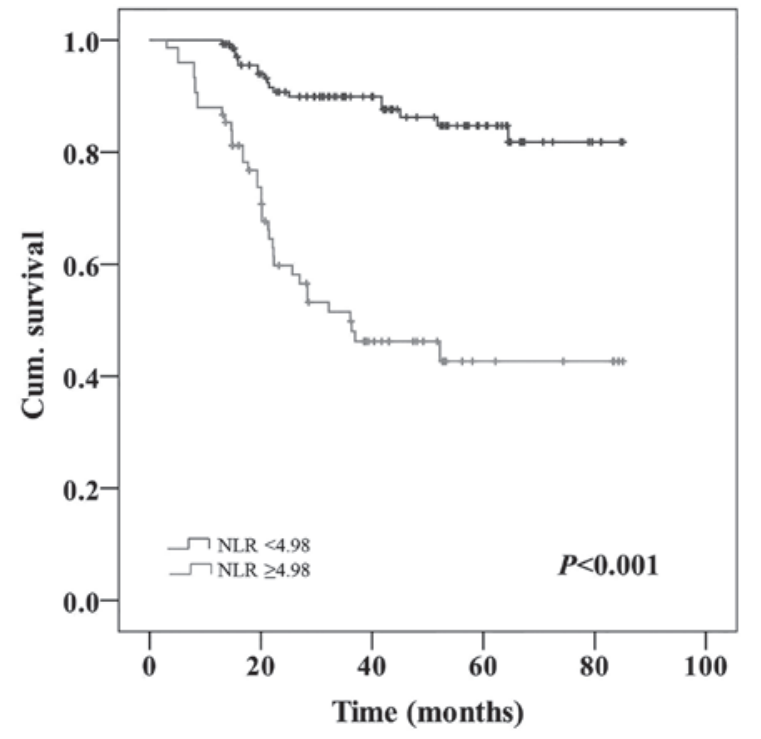

Figure 3. OS curve grouped by NLR. Patients with a high NLR $(\geq 4.98)$ possessed a significantly poorer OS time compared with patients with a low NLR (<4.98; P<0.001). OS, overal survival; NLR, neutrophil lymphocyte ratio; Cum., cumulative.

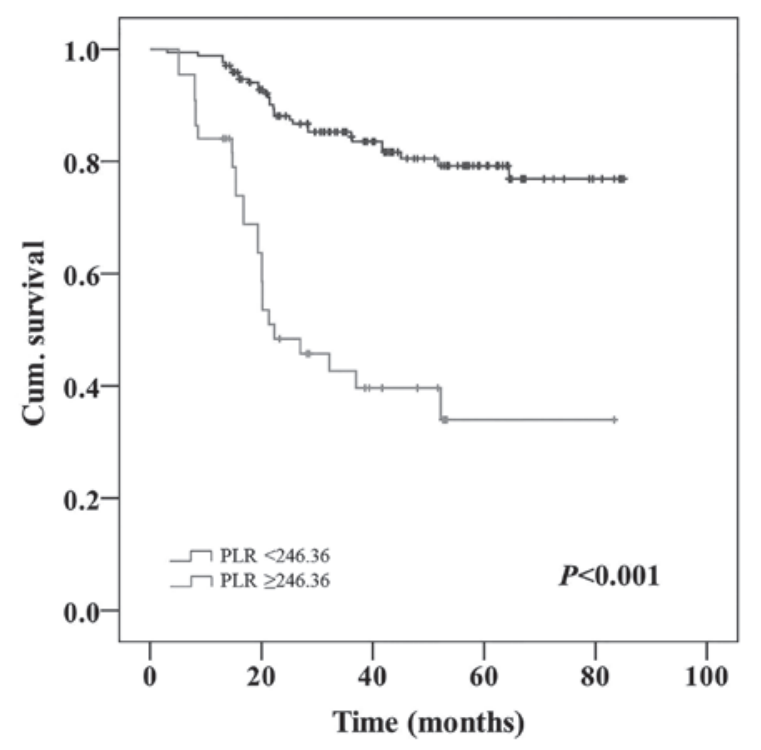

Figure 4. OS curve grouped by PLR. Patients with a high PLR $(\geq 246.36)$ possessed a significantly poorer OS time compared with patients with a low PLR (<246.36; P<0.001). OS, overal survival; PLR, platelet lymphocyte ratio; Cum., cumulative.

assessed in the pre-operative assessment $(12,14,18)$. Instead, NLR and PLR, calculated from leukocyte differential counts and platelet counts, respectively, are more readily available and inexpensive compared to CRP (7). Notably, numerous previous studies have already indicated that an elevated NLR and PLR is associated with a poorer survival in patients with ovarian (8), breast $(9,10)$, non-small cell lung (11), esophageal $(12,13)$, gastric $(7,14)$ and hepatocellular cancers $(15)$ and CRC $(2,16-18)$.

NLR is the ratio of the absolute neutrophil count to the absolute lymphocyte count, and therefore the association between a high NLR and a poor prognosis, as revealed in the

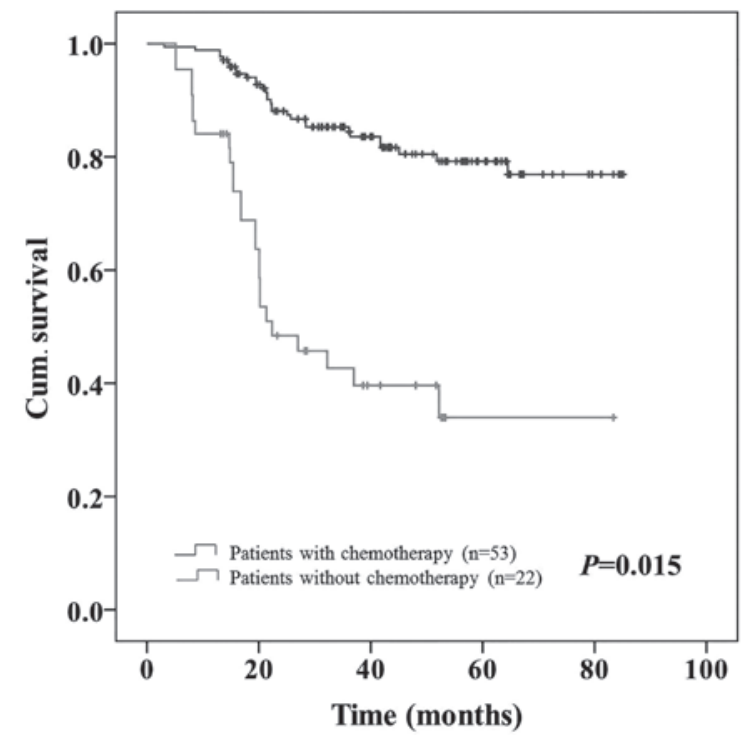

Figure 5. OS curve grouped by chemotherapy in patients with a high NLR. Adjuvant chemotherapy increased the 5-year OS rate from 29.2 to $49.1 \%$ in patients with a high NLR $(\geq 4.98 ; \mathrm{P}=0.015)$. OS, overall survival; NLR, neutrophil lymphocyte ratio; Cum., cumulative.

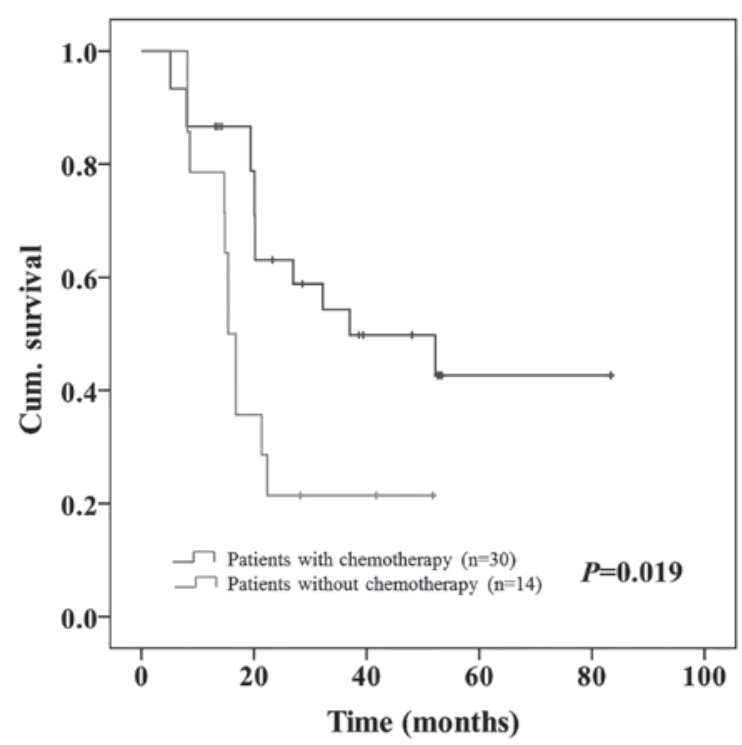

Figure 6. OS curve grouped by chemotherapy in patients with a high PLR. Adjuvant chemotherapy increased the 5-year OS rate from 21.4 to $42.7 \%$ in patients with a high PLR ( $\geq 246.36$; $P=0.019$ ). OS, overall survival; PLR, platelet lymphocyte ratio; Cum., cumulative.

present study, is possibly indicative of the tumor-promoting activity associated with neutrophilia in the tumor environment. Tumors are known to produce myeloid growth factors, including granulocyte colony-stimulating factor, tumor necrosis factor- $\alpha$, interleukin (IL)-1and IL-6, which may increase the number of neutrophilic granulocytes at the site of the tumor $(13,18)$. Neutrophilia promotes tumor growth and metastasis by remodeling the extracellular matrix and releasing reactive oxygen species, nitric oxide and arginase, which suppress the $\mathrm{T}$ cell response and increases the rate of mutagenesis (10). Additionally, neutrophilia suppresses lymphocyte activity, therefore counteracting the antitumor 
immune response (7). An elevated NLR has been associated with a poor survival rate in breast $(10)$, esophageal $(12,13)$ and gastric cancers $(7,14)$ and CRC $(26)$. Chiang et al demonstrated that patients with an elevated $\operatorname{NLR}(>3)$ in colon cancer appeared to possess larger tumors and a more advanced tumor stage, and patients with stages I-III CRC possessed a poorer 5-year disease-free survival rate (26).

PLR is the ratio of the absolute platelet count to absolute lymphocyte count, and therefore, the association of a high PLR with a poor prognosis, as revealed in the present study, is possibly indicative of the tumor-promoting activity associated with platelets. Platelets are known to be important in hemostasis and thrombosis (27). In addition, platelets mediate tumor cell growth, dissemination and angiogenesis (28). In turn, tumor cells induce platelet aggregation, which is known to be the trigger for the development of cancer-associated thrombosis (28). Platelets recruited to the tumor microenvironment consequently release platelet-derived growth factor and transforming growth factor to promote tumor growth (7,29). However, platelets also regulate angiogenesis by releasing numerous proangiogenic proteins, including vascular endothelial growth factor, and aid the maintenance of vascular integrity, therefore facilitating tumor cell survival and growth $(10,29)$. In addition, platelets shield tumor cells from host immune surveillance and direct cellular contact with natural killer cells by inducing platelet mimicry and constructing a mesh with fibrin that surrounds tumor cells within the vasculature during hematogenous dissemination (29). Tumor cells possess the ability to manipulate platelet activity to optimize tumor growth, proliferation, survival and metastasis (29). Several studies have identified the association between a poor survival rate and elevated PLR in solid tumors $(2,8,9,11,16,30)$. Szkandera et al revealed that an elevated PLR was significantly associated with a decreased time to recurrence and demonstrated a trend towards a decreased OS time in patients with stage II and III colon cancer that underwent curative resection (2). Liu et al reported that patients with CRC and a higher PLR possessed a significantly lower 5-year OS rate compared with patients with a low PLR, and identified pre-operative PLR as a clinically significant factor for the assessment of the prognosis of resectable CRC (16).

An elevated NLR or PLR is always accompanied by lymphopenia, which is caused by systemic inflammation and leads to the release of a number of inhibitory immunological mediators, particularly IL-10 and transforming growth factor- $\beta$. These inhibitory immunological mediators may exert an immunosuppressive effect with an impaired lymphocyte function (31).

The present study demonstrated that CRC patients with a high NLR or PLR tended to possess more clinicopathological factors associated with advanced disease, including poor tumor differentiation, the presence of a large tumor and a higher T classification. Regarding post-operative outcomes, in the present study, CRC patients with a high NLR or PLR possessed a significantly poorer 5-year OS rate compared with patients with a low NLR or PLR, particularly in patients with TNM stage II and III disease, indicating that NLR and PLR were effective independent prognostic factors. In addition, the present study aimed to identify which prognostic factor was the more effective. In this respect, 2 previous studies presented notable data. Kwon et al analyzed 200 patients that underwent curative resection and revealed that NLR and PLR were good prognostic biomarkers of OS rate in a univariate analysis, but only PLR was an independent prognostic factor in multivariate Cox proportional hazards analysis (18). He et al demonstrated that an elevated NLR, PLR and carcinoembryonic antigen (CEA) level were significant predictors of a poorer OS rate and progression-free survival time following first-line chemotherapy in patients with metastatic CRC, but only NLR and CEA were validated as independent factors (17). These two studies selected cutoff values for NLR and PLR from previous studies $(17,18)$. By contrast, the present study set the optimal cutoff value according to the maximal YI calculated from the ROC curve and grouped the patients accordingly, which is more suitable to the clinical data and more accurate for the specific study group. Additionally, the present study compared the predictive value of each factor using two methods.

Firstly, the present study compared NLR and PLR using their respective AUC values. According to the present results, the AUC for NLR was 0.748, which was greater compared with PLR (AUC=0.690). Secondly, the present study compared the risk of patients with a high NLR and PLR succumbing to CRC. The present results demonstrated that the risk of patients succumbing to $\mathrm{CRC}$ was increased in patients with a high NLR compared with patients with a high PLR (RR, 4.074 vs. 2.029). The AUC and RR data indicate that NLR is superior to PLR as a predictive factor for patients with CRC. Notably, Ishizuka et al investigated the prediction of cancer-specific survival time in patients with CRC using a parameter based on a combination of platelet count (COP) and NLR, and concluded that COP-NLR was a useful predictor of survival (32). Consequently, the present study also considered the combined effect of NLR and PLR on the prognostic significance. Accordingly, the present study allocated a score of 2,1 or 0 , according to the NLR and PLR, and performed a univariate analysis. The present results revealed that the combination of NLR and PLR was a valid prognostic factor.

The treatment history of the patients was another clinicopathological factor the present study investigated, although previous studies by Kwon et al and He et al did not consider the effect of an elevated NLR or PLR on adjuvant chemotherapy $(17,18)$. According to the present results, patients with a high NLR or PLR that accepted adjuvant chemotherapy possessed a significantly improved 5-year OS rate compared with patients that did not possess an elevated NLR or PLR and did not accept adjuvant chemotherapy $(\mathrm{P}=0.015$ and $\mathrm{P}=0.019$, respectively). This difference was not observed in patients with a low NLR or PLR. Therefore, adjuvant chemotherapy appeared to be more effective in CRC patients with a high NLR or PLR.

In conclusion, the present study validates the use of pre-operative NLR and PLR as independent prognostic factors for CRC patients. Notably, NLR was observed to be more effective than PLR for predicting CRC. In addition, the present data suggests that neutrophils and platelets are important in promoting CRC progression, but neutrophils are more crucial. Furthermore, adjuvant chemotherapy appeared 
to be more effective in CRC patients with a high NLR or PLR. However, as an observational, single hospital, small-scale study, the present study is limited. Larger prospective studies are required to confirm these preliminary results.

\section{Acknowledgements}

The present study was supported by the Natural Science Foundation of China (grant no. 61170123) and Natural Science Foundation of Hainan Province (grant no. 813226).

\section{References}

1. Ferlay J, Soerjomataram I, Dikshit R, Eser S, Mathers C, Rebelo M, Parkin DM, Forman D and Bray F: Cancer incidence and mortality worldwide: Sources, methods and major patterns in GLOBOCAN 2012. Int J Cancer 136: E359-E386, 2015.

2. Szkandera J, Pichler M, Absenger G, Stotz M, Arminger F, Weissmueller M, Schaberl-Moser R, Samonigg H, Kornprat P, Stojakovic T, et al: The elevated preoperative platelet to lymphocyte ratio predicts decreased time to recurrence in colon cancer patients. Am J Surg 208: 210-214, 2014.

3. Balkwill $\mathrm{F}$ and Mantovani A: Inflammation and cancer: Back to Virchow? Lancet 357: 539-545, 2001.

4. Grivennikov SI, Greten FR and Karin M: Immunity, inflammation, and cancer. Cell 140: 883-899, 2010.

5. Coussens LM and Werb Z: Inflammation and cancer. Nature 420: $860-867,2002$

6. DeNardo DG and Coussens LM: Inflammation and breast cancer. Balancing immune response: Crosstalk between adaptive and innate immune cells during breast cancer progression. Breast Cancer Res 9: 212, 2007.

7. Lee S, Oh SY, Kim SH, Lee JH, Kim MC, Kim KH and Kim HJ: Prognostic significance of neutrophil lymphocyte ratio and platelet lymphocyte ratio in advanced gastric cancer patients treated with FOLFOX chemotherapy. BMC Cancer 13: 350, 2013

8. Asher V, Lee J, Innamaa A and Bali A: Preoperative platelet lymphocyte ratio as an independent prognostic marker in ovarian cancer. Clin Transl Oncol 13: 499-503, 2011.

9. Krenn-Pilko S, Langsenlehner U, Thurner EM, Stojakovic T, Pichler M, Gerger A, Kapp KS and Langsenlehner T: The elevated preoperative platelet-to-lymphocyte ratio predicts poor prognosis in breast cancer patients. Br J Cancer 110: 2524-2530, 2014.

10. Azab B, Shah N, Radbel J, Tan P, Bhatt V, Vonfrolio S, Habeshy A, Picon A and Bloom S: Pretreatment neutrophil/lymphocyte ratio is superior to platelet/lymphocyte ratio as a predictor of long-term mortality in breast cancer patients. Med Oncol 30: $432,2013$.

11. Liu H, Wu Y, Wang Z, Yao Y, Chen F, Zhang H, Wang Y and Song Y: Pretreatment platelet-to-lymphocyte ratio (PLR) as a predictor of response to first-line platinum-based chemotherapy and prognosis for patients with non-small cell lung cancer. J Thorac Dis 5: 783-789, 2013.

12. Feng JF, Huang Y and Chen QX: Preoperative platelet lymphocyte ratio (PLR) is superior to neutrophil lymphocyte ratio (NLR) as a predictive factor in patients with esophageal squamous cell carcinoma. World J Surg Oncol 12: 58, 2014.

13. Feng JF, Huang Y, Zhao Q and Chen QX: Clinical significance of preoperative neutrophil lymphocyte ratio versus platelet lymphocyte ratio in patients with small cell carcinoma of the esophagus. ScientificWorldJournal 2013: 504365, 2013.

14. Jiang N, Deng JY, Liu Y, Ke B, Liu HG and Liang H: The role of preoperative neutrophil-lymphocyte and platelet-lymphocyte ratio in patients after radical resection for gastric cancer. Biomarkers 19: 444-451, 2014
15. Lai Q, Castro Santa E, Rico Juri JM, Pinheiro RS and Lerut J: Neutrophil and platelet-to-lymphocyte ratio as new predictors of dropout and recurrence after liver transplantation for hepatocellular cancer. Transpl Int 27: 32-41, 2014.

16. Liu H, Du X, Sun P, Xiao C, Xu Y and Li R: Preoperative platelet-lymphocyte ratio is an independent prognostic factor for resectable colorectal cancer. Nan Fang Yi Ke Da Xue Xue Bao 33: 70-73, 2013 (In Chinese).

17. He W, Yin C, Guo G, Jiang C, Wang F, Qiu H, Chen X, Rong R, Zhang $\mathrm{B}$ and $\mathrm{Xia} \mathrm{L}$ : Initial neutrophil lymphocyte ratio is superior to platelet lymphocyte ratio as an adverse prognostic and predictive factor in metastatic colorectal cancer. Med Oncol 30: 439, 2013

18. Kwon HC, Kim SH, Oh SY, Lee S, Lee JH, Choi HJ, Park KJ, Roh MS, Kim SG, Kim HJ and Lee JH: Clinical significance of preoperative neutrophil-lymphocyte versus platelet-lymphocyte ratio in patients with operable colorectal cancer. Biomarkers 17: 216-222, 2012

19. Edge SB, Byrd DR, Compton CC, Fritz AG, Greene FL and Trotti A (eds): AJCC Cancer Staging Manual. 7th edition. Springer, New York, NY, pp143-164, 2010.

20. National Comprehensive Cancer Network: NCCN Clinical Practice Guidelines in Oncology: Colon Cancer. Version 1. National Comprehensive Cancer Network, Washington, 2015.

21. National Comprehensive Cancer Network: NCCN Clinical Practice Guidelines in Oncology: Rectal Cancer. Version 1. National Comprehensive Cancer Network, Washington, 2015.

22. Zhao P, Dai M, Chen W and Li N: Cancer trends in China. Jpn J Clin Oncol 40: 281-285, 2010

23. Waldner MJ and Neurath MF: Colitis-associated cancer: The role of $\mathrm{T}$ cells in tumor development. Semin Immunopathol 31: 249-256, 2009.

24. Kwon KA, Kim SH, Oh SY, Lee S, Han JY, Kim KH, Goh RY, Choi HJ, Park KJ, Roh MS, et al: Clinical significance of preoperative serum vascular endothelial growth factor, interleukin-6, and C-reactive protein level in colorectal cancer. BMC Cancer 10: 203, 2010 .

25. Kim DK, Oh SY, Kwon HC, Lee S, Kwon KA, Kim BG, Kim SG, Kim SH, Jang JS, Kim MC, et al: Clinical significances of preoperative serum interleukin- 6 and C-reactive protein level in operable gastric cancer. BMC Cancer 9: 155, 2009.

26. Chiang SF, Hung HY, Tang R, Changchien CR, Chen JS, You YT, Chiang JM and Lin JR: Can neutrophil-to-lymphocyte ratio predict the survival of colorectal cancer patients who have received curative surgery electively? Int J Colorectal Dis 27: 1347-1357, 2012.

27. Nieswandt B, Aktas B, Moers A and Sachs UJ: Platelets in atherothrombosis: Lessons from mouse models. J Thromb Haemost 3: 1725-1736, 2005.

28. Goubran HA, Stakiw J, Radosevic M and Burnouf T: Platelets effects on tumor growth. Semin Oncol 41: 359-369, 2014.

29. Sharma D, Brummel-Ziedins KE, Bouchard BA and Holmes CE: Platelets in tumor progression: A host factor that offers multiple potential targets in the treatment of cancer. J Cell Physiol 229: 1005-1015, 2014.

30. Templeton AJ, Ace O, McNamara MG, Al-Mubarak M, Vera-Badillo FE, Hermanns T, Seruga B, Ocaña A, Tannock IF and Amir E: Prognostic role of platelet to lymphocyte ratio in solid tumors: A systematic review and meta-analysis. Cancer Epidemiol Biomarkers Prev 23: 1204-1212, 2014.

31. Salazar-Onfray F, López MN and Mendoza-Naranjo A: Paradoxical effects of cytokines in tumor immune surveillance and tumor immune escape. Cytokine Growth Factor Rev 18: 171-182, 2007.

32. Ishizuka M, Nagata H, Takagi K, Iwasaki Y and Kubota K: Combination of platelet count and neutrophil to lymphocyte ratio is a useful predictor of postoperative survival in patients with colorectal cancer. Br J Cancer 109: 401-407, 2013. 